\title{
Angle Calculations for a Five-Circle Diffractometer used in Surface X-ray Diffraction Experiments
}

\author{
By S.-K. Wang, P. Dai and H. Taub \\ Department of Physics and Astronomy, University of Missouri-Columbia, Columbia, Missouri 65211, USA
}

(Received 21 December 1992; accepted 8 April 1993)

\begin{abstract}
A straightforward method is presented for calculating the angle settings of a five-circle diffractometer used in surface diffraction experiments at synchrotron sources. Unlike the conventional four-circle unit, the five-circle diffractometer considered here allows detector motion perpendicular to the vertical scattering plane. The calculation method described here differs from previous ones for other diffractometers by resolving both the vector $\mathbf{G}$ to be accessed in the sample's reciprocal space and the wave-vector transfer $\mathbf{Q}$ into components parallel and perpendicular to a surface of interest. These vector components of $\mathbf{G}$ are a convenient means of describing the sample's initial orientation in the laboratory coordinate system. The corresponding components of $\mathbf{Q}$ allow two constraints on the diffraction condition to be simply expressed in the laboratory frame used throughout the calculations. One constraint is considered from each of the two sets: (1) fixing the incident or exit angle of the $\mathrm{X}$-ray beam with respect to the surface of interest; and (2) fixing the angle that the exit beam makes with the vertical scattering plane or requiring either the diffraction rod of interest or the normal to the cut surface of the crystal to remain in the horizontal plane. This method of calculating the angle settings can also be applied to other five-circle diffractometers as well as to four-circle diffractometers.
\end{abstract}

\section{Introduction}

Over the past decade, X-ray diffraction using synchrotron radiation has developed rapidly as a technique for characterizing the structures of surfaces, interfaces and adsorbed films. As discussed in recent reviews (Feidenhans'l, 1989; Robinson, 1991), the technique offers the advantage of kinematic analysis of the diffracted intensities while providing exceptionally high resolution in reciprocal space and sufficient sensitivity to probe a single layer of atoms.

The application of synchrotron X-ray diffraction to surface-structure studies has made new demands on both sample environments and diffractometers. Ultra-high-vacuum (UHV) chambers may be required

(C) 1993 International Union of Crystallography

Printed in Great Britain - all rights reserved for preparing and maintaining the surface of interest during the X-ray experiment. The need to rotate the sample under UHV conditions and to have various auxiliary surface-analytical probes and samplepreparation facilities near the $X$-ray scattering position has led to a variety of chamber designs, as reviewed by Feidenhans'l (1989). In some cases, it has been necessary to alter the conventional four-circle $\mathrm{X}$-ray diffractometer in use at many synchrotron beam lines in order to accommodate these chambers. Two examples of such modifications are the ' $z$-axis' (Brennan \& Eisenberger, 1984) and five-circle (Vlieg, Van't Ent, De Jongh, Neerings \& Van der Veen, 1987) diffractometers.

Another motive for modifying the conventional four-circle diffractometer for use in surface-diffraction experiments at synchrotrons is that it may be desirable to impose more than one constraint on the scattering geometry. With a four-circle instrument, only one constraint can be imposed because two of the sample's three rotational degrees of freedom are needed to satisfy the diffraction condition. The one additional rotational degree of freedom of the sample is usually used to fix the incident or exit angle of the X-ray beam with respect to the surface of interest (or to require the two angles to be equal). However, there are other constraints that might be useful in surface diffraction experiments, such as confining a diffraction rod to the horizontal plane in which the divergence of the incident beam is maximum. This allows one to utilize the finite instrumental resolution more effectively. An additional constraint of this type can be satisfied by introducing another degree of freedom into the diffractometer design, such as the table motion of the five-circle instrument.

In this paper, we consider a five-circle diffractometer similar to that treated by Vlieg, Van't Ent et al. (1987). In our case, the four-circle diffractometer is modified by mounting the detector on a rotary table that moves on a linear drive perpendicular to the vertical scattering plane, as shown in Fig. 1. In their case, the entire four-circle diffractometer is mounted on a table, effectively providing out-of-plane motion of the incident beam instead of the detector. Thus, the two five-circle instruments differ only in the direction

Journal of Applied Crystallography ISSN 0021-8898 $\quad$ (C) 1993 
traversed by the photons. To distinguish between them, we will refer to ours as the 'rotating-detector' five-circle diffractometer and theirs as the 'rotatingtable' five-circle diffractometer.

Our five-circle diffractometer was designed for use with an UHV chamber constructed for the investigation of physisorbed and other weakly bound films on single-crystal substrates (Dennison, Wang, Dai, Angot, Taub \& Ehrlich, 1992). The chamber is mounted on a conventional four-circle Huber 5020 diffractometer at the MATRIX (Midwest Analytical Team for Research Instrumentation of X-rays) beam line X18A at the National Synchrotron Light Source (NSLS) at Brookhaven National Laboratory. Owing to the size and configuration of the chamber, the region of reciprocal space accessible with the four-circle diffractometer is limited; in particular, it is impossible to perform specular reflectivity scans. The rotating-detector five-circle diffractometer addresses these shortcomings and allows diffraction scans under more than one constraint.

Here, we present calculations of the angle settings of the five-circle diffractometer when two scattering constraints of interest in surface experiments are imposed on the diffraction condition. By the diffraction condition, we refer to the rotation of a reciprocal-lattice vector $\mathbf{G}$ of a sample by motion of the $\varphi, \chi$ and $\omega$ circles of the diffractometer (see Fig. 1) so that $\mathbf{G}$ coincides with the wave-vector transfer $\mathbf{Q}=\mathbf{K}_{f}-\mathbf{K}_{i}$, where the incident wave vector $\mathbf{K}_{i}$ is fixed and the final wave vector $\mathbf{K}_{f}$ is defined by the detector angles $\delta$ and $2 \theta^{\prime}$ (e.g. Warren, 1969). Note that $\mathbf{G}$ need not be a reciprocal-lattice vector of a bulk sample. It may, for example, be a point on a diffraction rod associated with the surface of interest. The two additional constraints must be imposed on the diffraction condition in order to determine uniquely

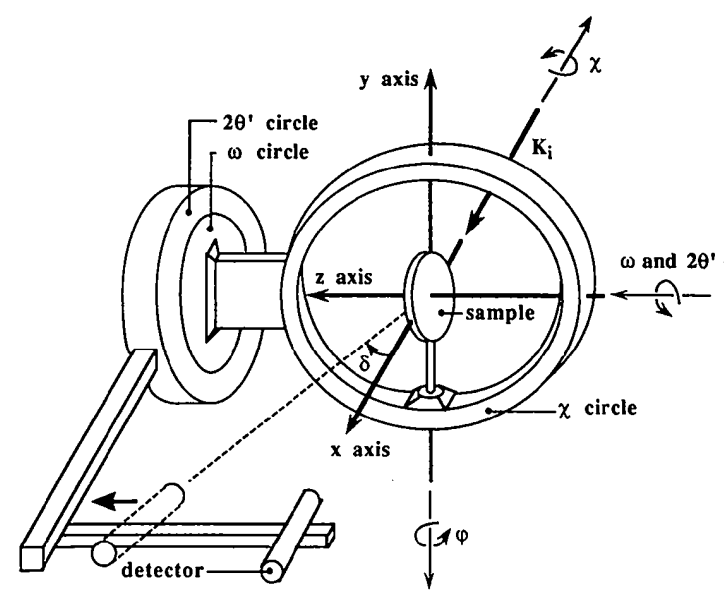

Fig. 1. The schematic diagram of a rotating-detector five-circle diffractometer when all the angle settings are zero. Arrows indicate the positive sense of rotation. the instrument's five angle settings. Such calculations are straightforward but, to our knowledge, have not yet been done for the rotating-detector five-circle diffractometer.

Busing \& Levy (1967) described a general method to calculate angle settings for three- and four-circle diffractometers. Their method was later adapted to surface diffraction scans in which the incident and exit angles that the X-ray beam makes with the surface of interest are controlled. Vlieg, Van der Veen, Macdonald \& Miller (1987) treated this problem for the rotating-table five-circle diffractometer and Mochrie (1988) treated it for the conventional fourcircle diffractometer. Earlier, Bloch (1985) derived an analogous scheme to operate a ' $z$-axis' instrument under the constraint of a fixed incident angle.

Because only two circles are required to rotate $\mathbf{G}$ into the direction of $\mathbf{Q}$ in the absence of constraints, there is an extra degree of rotational freedom with a four-circle diffractometer that allows this to be done in an unlimited number of ways. Busing \& Levy (1967) chose to express this extra degree of freedom in terms of $\psi$, the angle of rotation of the sample about $\mathbf{Q}$. In their subsequent calculations, Vlieg, Van der Veen, Macdonald \& Miller (1987) and Mochrie (1988) used a constraint on the incident or exit angle of the X-ray beam to determine $\psi$ uniquely.

Our approach differs from earlier ones in that we resolve the vectors $\mathbf{G}$ and $\mathbf{Q}$ into components parallel and perpendicular to a surface of interest. These vector components of $\mathbf{G}$ are a convenient means of describing the sample's initial orientation in the laboratory coordinate system. More importantly, the corresponding components of $\mathbf{Q}$ allow the constraints on the diffraction condition to be simply expressed in the laboratory coordinate system that is used throughout the calculations. This eliminates cumbersome transformations between coordinate systems fixed to each rotation axis of the diffractometer as in the previous angle-setting calculations based on the approach of Busing \& Levy (1967).

The remainder of the paper is organized as follows. We begin $\$ 2$ by defining the laboratory coordinate system and expressing the vectors $\mathbf{K}_{i}, \mathbf{K}_{f}$ and $\mathbf{Q}$ in it. Then, we distinguish between a sample's cut surface and a crystallographic plane of interest from which it may be slightly misaligned, define the components of $\mathbf{G}$ and $\mathbf{Q}$ parallel and perpendicular to this surface and plane and use these components to reformulate the angle-setting problem in $\S 3$. In $\S 4$, we calculate the angle settings of the rotating-detector five-circle diffractometer, assuming the components of $\mathbf{G}$ and $\mathbf{Q}$ parallel and perpendicular to the cut surface are known. $\S 5$ is concerned with calculating $\mathbf{Q}$ and its components parallel and perpendicular to the cut surface from various constraints of interest in surface diffraction experiments. In $\S 6$, we describe the 
procedure for determining both the orientation of the sample's cut surface and its crystallographic axes. Finally, application of the method to angle-setting calculations for four-circle and rotating-table fivecircle diffractometers is discussed briefly in $\$ 7$.

\section{Definition of the laboratory coordinate system}

As shown in Fig. 1, we define a right-handed Cartesian coordinate system fixed in the laboratory such that the positive $x$ axis is along the direction of the horizontal incident beam. The origin is at the center of the diffractometer where the sample is located. Note that our assignment of axes differs from that of Busing \& Levy (1967) in their Fig. 1(b). At $\omega=0$, the $\chi$ circle lies in the $y z$ plane so that it is perpendicular to the incident beam. In the four-circle diffractometer, the detector moves in the vertical $x y$ plane, making an angle $2 \theta$ (not shown in Fig. 1) with the incident beam. This avoids a near-zero polarization factor as the scattering angle $2 \theta$ approaches $90^{\circ}$.

In the rotating-detector five-circle diffractometer, the detector is mounted on a rotary table, which moves on a linear drive fixed to the detector arm and parallel to the $z$ axis of the laboratory system. The position of the detector arm is specified by the angle $2 \theta^{\prime}$ to distinguish it from $2 \theta$, which is the angle between $\mathbf{K}_{i}$ and $\mathbf{K}_{f}$. As shown in Fig. 2, $\delta$ is the angle between $\mathbf{K}_{f}$ and its projection onto the $x y$ plane. When $\delta$ and $2 \theta^{\prime}$ are both set to zero, the detector is in line with the incident beam. Arrows in Fig. 1 indicate the positive sense of rotation for each circle and of translation for the linear drive.

The need for the rotating-detector five-circle diffractometer in our experiments can now be explained more clearly. To perform specular reflection measurements with a four-circle diffractometer, the surface normal must be driven into the $x y$ plane. This requires a $90^{\circ}$ rotation of the $\chi$ circle, which is impossible with our UHV chamber mounted on the diffractometer. Moreover, the problem cannot be

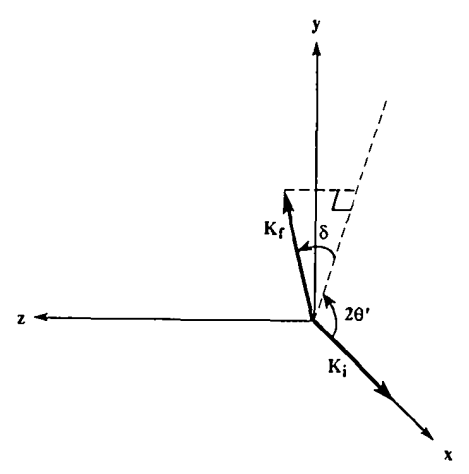

Fig. 2. The relation of $\mathbf{K}_{f}$ to the angles $2 \theta^{\prime}$ and $\delta$ in the laboratory coordinate system. Arrows indicate the positive sense of rotation. Detector is parallel to the direction of $\mathbf{K}_{f}$. solved by use of a table to rotate the entire diffractometer about the $y$ axis as with the five-circle diffractometer of Vlieg, Van der Veen et al. (1987), because our chamber does not allow sufficient range in the negative $\varphi$ direction (Dennison et al., 1992). However, by allowing the detector to move out of the $x y$ plane, specular-reflection measurements are feasible, as is access to larger values of $\mathbf{Q}_{\perp}$ in nonspecular scans. In $\S 4$, it is shown how this diffractometer also allows a second constraint to be imposed on the scattering geometry.

In the calculations below, it will be necessary to express the wave-vector transfer $\mathbf{Q}$ in the laboratory coordinate system. As shown in Fig. 2, the incident and diffracted wave vectors in the laboratory coordinate system are given by

$$
\mathbf{K}_{i}=k\left[\begin{array}{l}
1 \\
0 \\
0
\end{array}\right]
$$

and

$$
\mathbf{K}_{f}=k\left[\begin{array}{c}
\cos \delta \cos 2 \theta^{\prime} \\
\cos \delta \sin 2 \theta^{\prime} \\
\sin \delta
\end{array}\right]
$$

Therefore,

$$
\mathbf{Q}=\mathbf{K}_{f}-\mathbf{K}_{i}=k\left[\begin{array}{c}
\cos \delta \cos 2 \theta^{\prime}-1 \\
\cos \delta \sin 2 \theta^{\prime} \\
\sin \delta
\end{array}\right]=\left[\begin{array}{l}
Q_{x} \\
Q_{y} \\
Q_{z}
\end{array}\right],
$$

where $k=(2 \pi / \lambda)$ is the magnitude of the wave vector and $\lambda$ is the wavelength of the incident bcam. Using $Q^{2}=Q_{x}^{2}+Q_{y}^{2}+Q_{z}^{2}$ and (1), we have the following relation between the angles $2 \theta^{\prime}$ and $\delta$ :

$$
Q^{2}=2 k^{2}\left(1-\cos 2 \theta^{\prime} \cos \delta\right) \text {. }
$$

Note that $\cos 2 \theta^{\prime} \cos \delta=\cos 2 \theta$, where $2 \theta$ is the previously defined angle between $\mathbf{K}_{i}$ and $\mathbf{K}_{f}$.

\section{Reformulation of the problem}

Often there is more than one surface of interest in a diffraction experiment. One can cut a sample with the intention of exposing a surface parallel to one of the reflecting planes of the sample. However, in practice, this will usually result in some small angle of miscut between the unit vector $\mathbf{n}_{p}$ normal to the cut or 'physical' surface and $\mathbf{n}$, the unit vector normal to the reflecting (crystallographic) plane of interest. In the calculations below, we find it convenient to describe the initial sample orientation in terms of $\mathbf{G}_{\perp p}$ and $\mathbf{G}_{\| p}$, the components of $\mathbf{G}$ perpendicular and parallel to the cut surface, respectively, expressed in the laboratory coordinate system. Alternatively, we may wish to use $\mathbf{G}_{\perp}$ and $\mathbf{G}_{\|}$, the components of $\mathbf{G}$ perpendicular and parallel to the crystallographic plane of interest. 
To calculate $\mathbf{G}_{\perp p}\left(\mathbf{G}_{\perp}\right)$ and $\mathbf{G}_{\| p}\left(\mathbf{G}_{\|}\right)$, we need the components of $\mathbf{n}_{p}$ (n) in the laboratory coordinate system when the diffractometer is in its initial configuration $(\chi=\varphi=\omega=0$ in Fig. 1). The procedure for obtaining them is described in $\S 6$. Once $\mathbf{n}_{p}$ and $\mathbf{n}$ are known, it is a simple matter to obtain $\mathbf{G}_{\perp p}$, $\mathbf{G}_{\| p}, \mathbf{G}_{\perp}$ and $\mathbf{G}_{\|}$from

$$
\begin{aligned}
\mathbf{G}_{\perp p} & =\left(\mathbf{n}_{p} \cdot \mathbf{G}\right) \mathbf{n}_{p}, & \mathbf{G}_{\| \mathbf{p}} & =\mathbf{G}-\mathbf{G}_{\perp p}, \\
\mathbf{G}_{\perp} & =(\mathbf{n} \cdot \mathbf{G}) \mathbf{n}, & \mathbf{G}_{\|} & =\mathbf{G}-\mathbf{G}_{\perp} .
\end{aligned}
$$

As illustrated in Fig. 3(a), the components of $\mathbf{G}$ in (3) at the initial sample orientation can be used to define a right-handed orthogonal unit-vector triple $\mathbf{r}$, $\mathbf{s}, \mathbf{t}$ fixed to the sample:

$$
\mathbf{r}=\frac{\mathbf{G}_{\| p}}{G_{\| p}}, \quad \mathbf{t}=\frac{\mathbf{G}_{\perp p}}{G_{\perp p}}, \quad \mathbf{s}=\mathbf{t} \times \mathbf{r} .
$$

Another orthogonal unit-vector triple could be defined in a similar way based on the crystallographic plane of interest using (4).

As a result of the sample rotation necessary to satisfy the diffraction condition, the unit vectors $\mathbf{r}, \mathbf{s}$ and $\mathbf{t}$ will rotate into the vectors $\mathbf{r}^{\prime}, \mathbf{s}^{\prime}$ and $\mathbf{t}^{\prime}$, respectively, in the laboratory coordinate system. Similarly, the normal to the cut surface $\mathbf{n}_{p}$ and the normal to the crystallographic plane of interest $\mathbf{n}$ will become $\mathbf{n}_{p}^{\prime}$ and $\mathbf{n}^{\prime}$, respectively, after the sample rotation. We can then define the components of the wave-vector transfer $\mathbf{Q}$ perpendicular and parallel to the physical surface and crystallographic plane of interest after the sample rotation in analogy to (3) and (4):

$$
\begin{aligned}
\mathbf{Q}_{\perp p} & =\left(\mathbf{n}_{p}^{\prime} \cdot \mathbf{Q}\right) \mathbf{n}_{p}^{\prime}, & \mathbf{Q}_{\| p} & =\mathbf{Q}-\mathbf{Q}_{\perp p}, \\
\mathbf{Q}_{\perp} & =\left(\mathbf{n}^{\prime} \cdot \mathbf{Q}\right) \mathbf{n}^{\prime}, & \mathbf{Q}_{\|} & =\mathbf{Q}-\mathbf{Q}_{\perp} .
\end{aligned}
$$

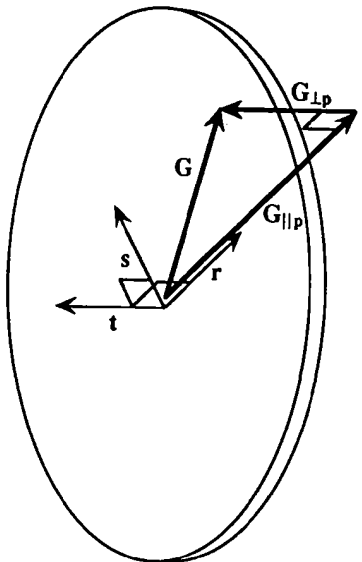

(a)

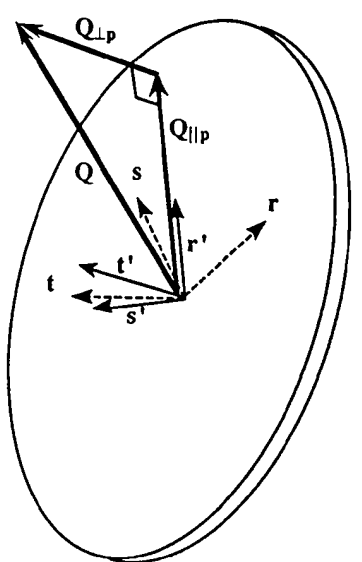

(b)
Fig. 3. (a) Diagram showing the orientation of the $\mathbf{r}, \mathbf{s}, \mathbf{t}$ orthogonal unit-vector triple in the initial samplc configuration. (b) Diagram showing the $\mathbf{r}^{\prime}, \mathbf{s}^{\prime}, \mathbf{t}^{\prime}$ orthogonal unit-vector triple after rotation of the sample to satisfy the diffraction condition; the unit vectors $\mathbf{r}, \mathbf{s}$ and $\mathbf{t}$ are shown by dashed lines for clarity.
Satisfaction of the diffraction condition requires

$$
\mathbf{r}^{\prime}=\frac{\mathbf{Q}_{\| p}}{Q_{\| p}}, \quad \mathbf{t}^{\prime}=\frac{\mathbf{Q}_{\perp p}}{Q_{\perp p}}, \quad \mathbf{s}^{\prime}=\mathbf{t}^{\prime} \times \mathbf{r}^{\prime},
$$

as shown in Fig. 3(b).

We can now reformulate the angle-settings problem as one of calculating values of the angles $\varphi, \chi$ and $\omega$ that will exactly superimpose the two unit-vector triples $\mathbf{r}, \mathbf{s}, \mathbf{t}$ and $\mathbf{r}^{\prime}, \mathbf{s}^{\prime}, \mathbf{t}^{\prime}$. Such a calculation was discussed by Busing \& Levy (1967) in obtaining the orientation matrix. The method we present in the next section differs from theirs in that we perform rotations of the sample in the laboratory coordinate system. We find this approach easier because the constraints of interest in surface-diffraction experiments are simply expressed in this frame.

\section{Calculation of the angle settings of the five-circle diffractometer for a specified surface orientation}

In the next section, we calculate $\mathbf{Q}$ and $\mathbf{Q}_{\perp p}$ (and hence $\mathbf{Q}_{\| p}$ ) in the laboratory coordinate system from the diffraction condition $Q=G$ and two additional constraints on the scattering geometry. In this section, we simply assume that $\mathbf{Q}$ and $\mathbf{Q}_{\perp p}$ are known and use them to obtain values of the diffractometer angles $\varphi$, $\chi, \omega, \delta$ and $2 \theta^{\prime}$. The special cases where either $\mathbf{Q}_{\perp p}=0$ (in-plane scattering) or $\mathbf{Q}_{\| p}=0$ (specular reflection) are treated in the Appendix.

We first note that the detector angles $\delta$ and $2 \theta^{\prime}$ can be calculated immediately from (1) if $\mathbf{Q}$ is known in the laboratory coordinate system. As discussed in the previous section, we calculate the sample rotation angles $\varphi, \chi$ and $\omega$ by requiring that the two unit-vector triples $\mathbf{r}, \mathbf{s}, \mathbf{t}$ and $\mathbf{r}^{\prime}, \mathbf{s}^{\prime}, \mathbf{t}^{\prime}$ superimpose. These sample rotations are accomplished by simultaneous motion of the $\varphi, \chi$ and $\omega$ drives, after which $\mathbf{G}$ will point in the same direction as $\mathbf{Q}$. Note that the $\varphi$ and $\chi$ axes will themselves move in the laboratory frame. However, because neither the $\chi$ axis moves as a result of the $\varphi$ rotation nor the $\omega$ axis as a result of the $\chi$ rotation, the process can also be regarded as successive rotations by angles $\varphi, \chi$ and $\omega$ represented by the noncommuting matrices $\Phi(\varphi), X(\chi)$ and $\Omega(\omega)$. These matrices, expressed in the laboratory coordinate system, are

$$
\begin{aligned}
& \Phi(\varphi)=\left[\begin{array}{ccc}
\cos \varphi & 0 & -\sin \varphi \\
0 & 1 & 0 \\
\sin \varphi & 0 & \cos \varphi
\end{array}\right], \\
& X(\chi)=\left[\begin{array}{ccc}
1 & 0 & 0 \\
0 & \cos \chi & \sin \chi \\
0 & -\sin \chi & \cos \chi
\end{array}\right], \\
& \Omega(\omega)=\left[\begin{array}{ccc}
\cos \omega & -\sin \omega & 0 \\
\sin \omega & \cos \omega & 0 \\
0 & 0 & 1
\end{array}\right] .
\end{aligned}
$$


The wave-vector transfer $\mathbf{Q}$ and the reciprocallattice vector $\mathbf{G}$ are related by

$$
\mathbf{Q}_{x y z}=S(\varphi, \chi, \omega) \mathbf{G}_{x y z}
$$

where

$$
\begin{aligned}
& S(\varphi, \chi, \omega) \\
& =\Omega(\omega) X(\chi) \Phi(\varphi) \\
& =\left[\begin{array}{lll}
s_{11} & s_{12} & s_{13} \\
s_{21} & s_{22} & s_{23} \\
s_{31} & s_{32} & s_{33}
\end{array}\right] \\
& =\left[\begin{array}{cc}
(\cos \omega \cos \varphi-\sin \omega \sin \chi \sin \varphi) & (-\sin \omega \cos \chi) \\
(\sin \omega \cos \varphi+\cos \omega \sin \chi \sin \varphi) & (\cos \omega \cos \chi) \\
(\cos \chi \sin \varphi) & (-\sin \chi)
\end{array}\right. \\
& \left.\begin{array}{c}
(-\cos \omega \sin \varphi-\sin \omega \sin \chi \cos \varphi) \\
(-\sin \omega \sin \varphi+\cos \omega \sin \chi \cos \varphi) \\
(\cos \chi \cos \varphi)
\end{array}\right] .
\end{aligned}
$$

$\mathbf{G}_{x y z}$ and $\mathbf{Q}_{x y z}$ are the vectors expressed in the $x y z$ laboratory coordinate system. For simplicity, we henceforth drop the subscript $x y z$ when denoting vectors in the laboratory frame. Note that the unitarity of the matrices $\Phi(\varphi), X(\chi)$ and $\Omega(\omega)$ ensures that $Q=G$. Similarly, we have $Q_{\perp p}=G_{\perp p}$.

Instead of trying to solve (12) directly for the angles $\varphi, \chi$ and $\omega$, we introduce matrices $T_{1}$ and $T_{2}^{-1}$, which transform any vector from the laboratory coordinate system to the rst coordinate system and from the $\mathbf{r}^{\prime} \mathbf{s}^{\prime} \mathbf{t}^{\prime}$ coordinate system back to the laboratory coordinate system, respectively. $T_{1}$ and $T_{2}^{-1}$ can be written as

$$
\begin{gathered}
T_{1}=\left[\begin{array}{lll}
\mathbf{r} \cdot \mathbf{x} & \mathbf{r} \cdot \mathbf{y} & \mathbf{r} \cdot \mathbf{z} \\
\mathbf{s} \cdot \mathbf{x} & \mathbf{s} \cdot \mathbf{y} & \mathbf{s} \cdot \mathbf{z} \\
\mathbf{t} \cdot \mathbf{x} & \mathbf{t} \cdot \mathbf{y} & \mathbf{t} \cdot \mathbf{z}
\end{array}\right]=\left[\begin{array}{lll}
(r)_{x} & (r)_{y} & (r)_{z} \\
(s)_{x} & (s)_{y} & (s)_{z} \\
(t)_{x} & (t)_{y} & (t)_{z}
\end{array}\right] \\
T_{2}^{-1}=\left[\begin{array}{lll}
\mathbf{x} \cdot \mathbf{r}^{\prime} & \mathbf{x} \cdot \mathbf{s}^{\prime} & \mathbf{x} \cdot \mathbf{t}^{\prime} \\
\mathbf{y} \cdot \mathbf{r}^{\prime} & \mathbf{y} \cdot \mathbf{s}^{\prime} & \mathbf{y} \cdot \mathbf{t}^{\prime} \\
\mathbf{z} \cdot \mathbf{r}^{\prime} & \mathbf{z} \cdot \mathbf{s}^{\prime} & \mathbf{z} \cdot \mathbf{t}^{\prime}
\end{array}\right]=\left[\begin{array}{lll}
\left(r^{\prime}\right)_{x} & \left(s^{\prime}\right)_{x} & \left(t^{\prime}\right)_{x} \\
\left(r^{\prime}\right)_{y} & \left(s^{\prime}\right)_{y} & \left(t^{\prime}\right)_{y} \\
\left(r^{\prime}\right)_{z} & \left(s^{\prime}\right)_{z} & \left(t^{\prime}\right)_{z}
\end{array}\right] .
\end{gathered}
$$

These matrices allow us to express the relation between $\mathbf{G}$ and $\mathbf{Q}$ as follows:

$$
\mathbf{Q}=T_{2}^{-1} \mathbf{Q}_{r^{\prime} s^{\prime}}=T_{2}^{-1} \mathbf{G}_{r s t}=T_{2}^{-1} T_{1} \mathbf{G} .
$$

Here, $\mathbf{Q}_{r^{\prime} s^{\prime} t^{\prime}}$ and $\mathbf{G}_{r s t}$ are vectors expressed in the $\mathbf{r}^{\prime} \mathbf{s}^{\prime} \mathbf{t}^{\prime}$ and rst coordinate systems, respectively. They are equal because the components of $\mathbf{G}$ in a coordinate system fixed to the sample do not change upon sample rotation; i.e. $\mathbf{G}_{r s t}=\mathbf{G}_{r^{\prime} s^{\prime} t^{\prime}}$ and $\mathbf{G}_{r^{\prime} t^{\prime} t^{\prime}}=\mathbf{Q}_{r^{\prime} s^{\prime} t^{\prime}}$ by the diffraction condition. Comparing (16) with (12), we obtain

$$
S(\varphi, \chi, \omega)=T_{2}^{-1} T_{1} \equiv T_{3} .
$$

From (13) and (17), it is straightforward to calculate the rotation angles $\varphi, \chi$ and $\omega$. For $\chi$, we have

$$
\chi=\arcsin \left[-\left(T_{3}\right)_{32}\right] \text {, }
$$

where $\left(T_{3}\right)_{32}$ is the element in the third row and second column of the matrix $T_{3}$. This equation yields a unique value for $\chi$ in the range $-\pi / 2 \leq \chi \leq \pi / 2$. To calculate $\varphi$, we use the relations $\left(T_{3}\right)_{31}=\cos \chi \sin \varphi$ and $\left(T_{3}\right)_{33}=\cos \chi \cos \varphi$, so that

$$
\varphi=\arctan \left[\left(T_{3}\right)_{31} /\left(T_{3}\right)_{33}\right] .
$$

Similarly, we have the following relations for $\omega$ : $\left(T_{3}\right)_{12}=-\sin \omega \cos \chi$ and $\left(T_{3}\right)_{22}=\cos \omega \cos \chi$, so that

$$
\omega=\arctan \left[-\left(T_{3}\right)_{12} /\left(T_{3}\right)_{22}\right] .
$$

This completes the calculation of the three samplerotation angles $\varphi, \chi$ and $\omega$.

\section{Calculation of $Q, Q_{\perp p}$ and $Q_{\| p}$ for the five-circle diffractometer under various constraints}

In the last section, we assumed knowledge of $\mathbf{Q}$ and its components $\mathbf{Q}_{\perp p}$ and $\mathbf{Q}_{\| p}$ with respect to the cut surface after rotation of the sample to satisfy the diffraction condition. These vectors, along with $\mathbf{G}_{\perp p}$ and $\mathbf{G}_{\| p}$, the components of the reciprocal-lattice vector of interest at the initial sample orientation in the laboratory frame, were sufficient to determine uniquely the angle settings of the rotating-detector five-circle diffractometer. In this section, we calculate $\mathbf{Q}$ and $\mathbf{Q}_{\perp p}$ in the laboratory coordinate system for various constraints of interest in surface diffraction experiments.

The first constraint that we impose on the scattering geometry is to fix either the incident or exit angle of the X-ray beam with respect to the surface of interest. One might instead require the two angles to be equal (Robinson, 1988). In the discussion above, we have already selected the cut surface as the one of interest. For this surface, the incident angle $\alpha$ and exit angle $\beta$ are related by $G_{\perp p}=Q_{\perp p}=k(\sin \alpha+\sin \beta)$, as can be seen from Fig. $4(a)$. Hence, the first constraint can always be expressed by specifying the incident angle $\alpha$ (Vlieg, Van der Veen et al., 1987; Mochrie, 1988). We can see from Fig. $4(b)$ that fixing $\alpha$ determines the $x$ component of $\mathbf{Q}_{\perp p}$ in the laboratory coordinate system:

$$
\begin{aligned}
\left(Q_{\perp p}\right)_{x}=\mathbf{Q}_{\perp p} \cdot \mathbf{K}_{i} / K_{i} & =Q_{\perp p} \cos [(\pi / 2)+\alpha] \\
& =-G_{\perp p} \sin \alpha .
\end{aligned}
$$

With the out-of-plane motion of the detector in the five-circle diffractometer, a second constraint must be imposed to yield a unique solution for the angle settings. Two useful choices for this constraint have been discussed by Vlieg, Van der Veen et al. (1987) for a five-circle diffractometer. At the diffraction 
condition, either (1) the cut-surface normal $\mathbf{n}_{p}^{\prime}$ or (2) the crystallographic-plane normal $\mathbf{n}^{\prime}$ lie in the horizontal $(x z)$ plane. The condition on $\mathbf{n}_{p}^{\prime}$ ensures that, for small angles of incidence, the sample intercepts as much of the incident beam as possible while that on $n^{\prime}$ allows one to utilize the finite instrumental resolution more effectively.

The relation between the direction of the crystallographic plane normal $\mathbf{n}^{\prime}$ and the instrumental resolution can be seen as follows. In a surface scattering experiment, one may be interested in scanning a diffraction rod that is parallel to $\mathbf{n}^{\prime}$. As discussed by Vlieg, Van der Veen et al. (1987), the intensity distribution along the rod is generally broad, but it can be narrow perpendicular to the rod (parallel to the crystallographic plane). For this reason, it is desirable to have better $Q$ resolution in a direction perpendicular to the rod than parallel to it. Since the divergence of a beam from a synchrotron source is much larger horizontally than vertically, this can be achieved by confining the diffraction rod to the horizontal plane.

A third choice of constraint for the five-circle diffractometer is simply to fix $\delta$, the angle between $\mathbf{K}_{f}$ and the $x y$ plane (see Fig. 2). One reason for doing this is to prevent a reduction in scattered intensity at large $\delta$ from the polarization factor (e.g. Warren, 1969). Another reason for fixing $\delta$ arises when the divergence of the exit beam is determined by a pair of slits. Then the $Q$ resolution of the diffractometer depends on the sample-slit distance and hence on $\delta$.

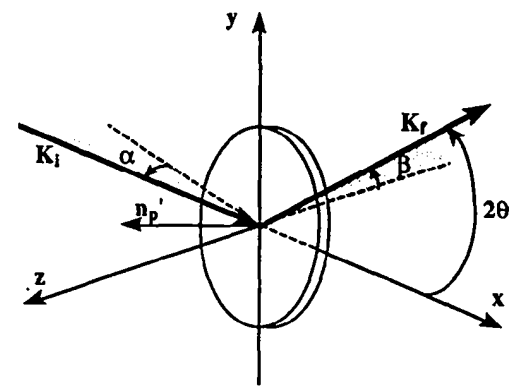

(a)

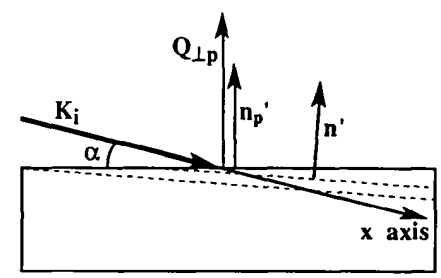

(b)

Fig. 4. (a) Real-space scattering geometry: the incident beam of wave vector $\mathbf{K}_{i}$ is directed along the $+x$ axis and makes an angle $2 \theta$ with the outgoing wave vector $\mathbf{K}_{f} ; \alpha$ and $\beta$ are the angles of the incident and diffracted beams, respectively, to the sample surface. $(b)$ The relation between $\mathbf{K}_{i}$, the cut surface, and the crystallographic plane of interest after the sample rotation. The dashed lines represent the crystallographic plane.
In this case, it may be useful to fix $\delta$ in order not to affect the instrumental resolution.

We now show how to obtain $\mathbf{Q}$ and the $y$ and $z$ components of $\mathbf{Q}_{\perp p}$ in the laboratory frame for each of these three choices of the second constraint.

(i) The detector rotation angle $\delta$ is held constant. For fixed $\delta$, one can easily calculate $2 \theta^{\prime}$ from (2) and obtain $\mathbf{Q}$ from (1). To facilitate calculation of the $y$ and $z$ components of $\mathbf{Q}_{\perp p}$, we introduce a new Cartesian coordinate system spanned by the orthogonal unit-vector triple $\mathbf{x}^{1}, \mathbf{y}^{1}, \mathbf{z}^{1}$, in which the $z$ component of $\mathbf{Q}$ is zero:

$$
\begin{aligned}
\mathbf{x}^{1} & =\mathbf{x}, \\
\mathbf{y}^{1} & =\left[Q_{y} /\left(Q_{y}^{2}+Q_{z}^{2}\right)^{1 / 2}\right] \mathbf{y}+\left[Q_{z} /\left(Q_{y}^{2}+Q_{z}^{2}\right)^{1 / 2}\right] \mathbf{z} \\
& =\xi \mathbf{y}+\zeta \mathbf{z}, \\
\mathbf{z}^{1} & =\mathbf{x}^{1} \times \mathbf{y}^{1} .
\end{aligned}
$$

In this coordinate system, the calculation of $\mathbf{Q}_{\perp p}$ is no more difficult than for the four-circle diffractometer where $\mathbf{Q}$ is confined to the $x y$ plane in the laboratory frame.

Next, define the matrix $T_{4}$ such that $T_{4}^{-1}$ transforms any vector from the $\mathbf{x}^{1} \mathbf{y}^{1} \mathbf{z}^{1}$ to the $\mathbf{x y z}$ system:

$$
T_{4}^{-1}=\left[\begin{array}{lll}
\mathbf{x} \cdot \mathbf{x}^{1} & \mathbf{x} \cdot \mathbf{y}^{1} & \mathbf{x} \cdot \mathbf{z}^{1} \\
\mathbf{y} \cdot \mathbf{x}^{1} & \mathbf{y} \cdot \mathbf{y}^{1} & \mathbf{y} \cdot \mathbf{z}^{1} \\
\mathbf{z} \cdot \mathbf{x}^{1} & \mathbf{z} \cdot \mathbf{y}^{1} & \mathbf{z} \cdot \mathbf{z}^{1}
\end{array}\right]=\left[\begin{array}{rrr}
1 & 0 & 0 \\
0 & \xi & -\zeta \\
0 & \zeta & \xi
\end{array}\right] .
$$

In particular, we can apply $T_{4}^{-1}$ to $\mathbf{Q}_{x^{\prime} y^{\prime} z^{1}}$ and its components:

$$
\begin{gathered}
\mathbf{Q}=T_{4}^{-1} \mathbf{Q}_{x^{\prime} y^{\prime} z^{1}}, \quad \mathbf{Q}_{\perp p}=T_{4}^{-1}\left(\mathbf{Q}_{\perp p}\right)_{x^{1} y^{\prime} z^{1},} \\
\mathbf{Q}_{\| p}=T_{4}^{-1}\left(\mathbf{Q}_{\| p}\right)_{x^{\prime} y^{1} z^{1} .}
\end{gathered}
$$

Because $\mathbf{Q}$ has already been determined, $\mathbf{Q}_{x^{1} y^{1} z^{1}}$ can be calculated to be

$$
\mathbf{Q}_{x^{\prime} y^{\prime} z^{1}}=\left(\begin{array}{l}
a_{1} \\
a_{2} \\
a_{3}
\end{array}\right)=\left(\begin{array}{c}
Q_{2} \\
\left(Q_{y}^{2}+Q_{z}^{2}\right)^{1 / 2} \\
0
\end{array}\right),
$$

as can be seen from (22). Now define the components of $\mathbf{Q}_{\perp p}$ and $\mathbf{Q}_{\| p}$ in the $\mathbf{x}^{1} \mathbf{y}^{1} \mathbf{z}^{1}$ coordinate system to be

$$
\left(\mathbf{Q}_{\perp p}\right)_{x^{\prime} y^{\prime} z^{1}}=\left(\begin{array}{c}
b_{1} \\
b_{2} \\
b_{3}
\end{array}\right), \quad\left(\mathbf{Q}_{\| p}\right)_{x^{\prime} y^{\prime} z^{1}}=\left(\begin{array}{c}
c_{1} \\
c_{2} \\
c_{3}
\end{array}\right) .
$$

Note that $b_{1}$ is already known from the first constraint $\left[(21)\right.$, because $\left(Q_{\perp p}\right)_{x}=\left(Q_{\perp p}\right)_{x^{1}}$ from (24). To find $b_{2}$ and $b_{3}$, we use the fact that $\left(\mathbf{Q}_{\| p}\right)_{x^{1} y^{1} z^{1}},\left(\mathbf{Q}_{\perp p}\right)_{x^{\prime} y^{1} z^{\prime}}$ and $\mathbf{Q}_{x^{\prime} y^{\prime} z^{\prime}}$ form a right triangle so that

$$
\begin{aligned}
\mathbf{Q}_{x^{\prime} y^{\prime} z^{\prime}} \cdot\left(\mathbf{Q}_{\perp p}\right)_{x^{\prime} y^{\prime} z^{\prime}} & =\left|\left(\mathbf{Q}_{\perp p}\right)_{x^{\prime} y^{\prime} z^{1}}\right|^{2} \\
& =a_{1} b_{1}+a_{2} b_{2}+a_{3} b_{3} \\
& =a_{1} b_{1}+a_{2} b_{2}
\end{aligned}
$$


because $a_{3}=0[(25)]$. Then, from (26) and (27), we obtain

$$
\left|\left(\mathbf{Q}_{\perp p}\right)_{x^{\prime} y^{\prime} z^{\prime}}\right|^{2}=b_{1}^{2}+b_{2}^{2}+b_{3}^{2}=a_{1} b_{1}+a_{2} b_{2}=G_{\perp p}^{2},
$$

where the last step follows from the unitarity of the matrix $S$ in (12) and of $T_{4}^{-1}$ in (23). Solving (28), we obtain $b_{2}$ and $b_{3}$ in terms of known quantities:

$$
\begin{gathered}
b_{2}=\left(G_{\perp p}^{2}-a_{1} b_{1}\right) / a_{2}, \\
b_{3}=\left(G_{\perp p}^{2}-b_{1}^{2}-b_{2}^{2}\right)^{1 / 2} .
\end{gathered}
$$

Therefore, we have calculated $\left(\mathbf{Q}_{\perp p}\right)_{x^{\prime} y^{\prime} z^{\prime}}$ and can obtain $\mathbf{Q}_{\perp p}=T_{4}^{-1}\left(\mathbf{Q}_{\perp}^{\prime}\right)_{x^{\prime} y^{\prime} z^{\prime}}$. Because $\mathbf{Q}$ has already been determined, $\mathbf{Q}_{\| p}$ can be obtained from $\mathbf{Q}_{\| p}=\mathbf{Q}-\mathbf{Q}_{\perp p \text {. }}$. Knowing $\mathbf{Q}_{\perp p}$ and $\mathbf{Q}_{\| p}$, we can calculate the unit vector triple $\mathbf{r}^{\prime}, \mathbf{s}^{\prime}, \mathbf{t}^{\prime}$ in (8) and hence the matrix $T_{3}$ in (17) needed in the calculation of the sample rotation angles $\varphi, \chi$ and $\omega$.

(ii) After rotation to the diffraction condition, the normal to the cut surface $\mathbf{n}_{p}^{\prime}$ remains in the horizontal plane. From Fig. 1, we see that this constraint requires that the $y$ component of $\mathbf{n}_{p}^{\prime}$ to be zero, i.e. $\left(n_{p}^{\prime}\right)_{y}=0$. Since $\mathbf{Q}_{\perp p}$ is parallel to $\mathbf{n}_{p}^{\prime}$, we also have $\left(Q_{\perp p}\right)_{y}=0$. Note that the expression of this constraint is much simpler than in a method based on the Busing \& Levy (1967) formalism [see equation (35) in Vlieg, Van der Veen et al. (1987)]. Recalling that $\left(Q_{\perp p}\right)_{x}$ is known from (21) and using the condition $Q_{\perp p}^{2}=G_{\perp p}^{2}$, we can solve for $\left(Q_{\perp p}\right)_{z}$ :

$$
\begin{aligned}
\left(Q_{\perp p}\right)_{z} & =\left[G_{\perp p}^{2}-\left(Q_{\perp p}\right)_{x}^{2}-\left(Q_{\perp p}\right)_{y}^{2}\right]^{1 / 2} \\
& =\left[G_{\perp p}^{2}-\left(Q_{\perp p}\right)_{x}^{2}\right]^{1 / 2} \\
& =G_{\perp p}\left[1-\sin ^{2} \alpha\right]^{1 / 2} .
\end{aligned}
$$

Here, the negative solution for $\left(Q_{\perp p}\right)_{z}$ is excluded because $\chi$ is constrained to the range $-\pi / 2 \leq \chi \leq \pi / 2$. Hence, we again have all three components of $\mathbf{Q}_{\perp p}$.

To obtain $\mathbf{Q}$, we first note that its $x$ component is known from (1) and (2):

$$
Q_{x}=-Q^{2} / 2 k
$$

where $Q^{2}=G^{2}$. Then, in analogy to (27), we have

$$
\begin{aligned}
\mathbf{Q} \cdot \mathbf{Q}_{\perp p} & =Q_{\perp p}^{2} \\
& =Q_{x}\left(Q_{\perp p}\right)_{x}+Q_{y}\left(Q_{\perp p}\right)_{y}+Q_{z}\left(Q_{\perp p}\right)_{z} \\
& =Q_{x}\left(Q_{\perp p}\right)_{x}+Q_{z}\left(Q_{\perp p}\right)_{z} \\
& =G_{\perp p}^{2},
\end{aligned}
$$

since $\left(Q_{\perp_{p}}\right)_{y}=0$. This equation can be solved for $Q_{z}$ to give

$$
Q_{z}=\left[G_{\perp p}^{2}-Q_{x}\left(Q_{\perp p}\right)_{x}\right] /\left(Q_{\perp p}\right)_{z},
$$

where all quantities are now known on the right-hand side. Thus, we have calculated both $Q_{x}$ and $Q_{z}$; knowing $Q=G$, we can find $Q_{y}$. This completes the determination of both $\mathbf{Q}_{\perp p}$ and $\mathbf{Q}$ in the laboratory frame.

(iii) After rotation to the diffraction condition, the normal to the crystallographic surface $\mathbf{n}^{\prime}$ remains in the horizontal plane. This constraint requires that the $y$ component of $\mathbf{n}^{\prime}$ be zero (see Fig. 1):

$$
\left(n^{\prime}\right)_{y}=\left(T_{3} \mathbf{n}\right)_{y}=0
$$

where $T_{3}$ has been defined in (17). Because $Q_{\perp}$ is parallel to $\mathbf{n}^{\prime},(35)$ implies $\left(Q_{\perp}\right)_{y}=0$. Note that the fixed-incident-angle constraint in (21) is with respect to the cut surface and yields $\left(Q_{\perp p}\right)_{x}$, whereas (35) involves a condition on $\left(Q_{\perp}\right)_{y}$. As in the case of the rotating-table five-circle diffractometer (Vlieg, Van der Veen et al., 1987), there is no simple analytical solution for this situation. However, a numerical solution can be found by varying $\delta$ until (35) is satisfied. As discussed in part (i) of this section, specifying $\delta$ determines $\mathbf{Q}$, which, together with the fixed-incidentangle constraint in (21), determines $\mathbf{Q}_{\perp p}$ and $\mathbf{Q}_{\| p}$. These components of $\mathbf{Q}$ and those of $\mathbf{G}$ in the initial sample orientation yield a unique $T_{3}$. If the miscut angle is small, an initial value for $\delta$ can be obtained by using constraint (ii). For samples with no miscut, constraints (ii) and (iii) are equivalent.

The calculations in this section could be repeated considering a crystallographic plane rather than the cut surface to be of interest. The problem would then be to obtain $\mathbf{Q}_{\perp}$ defined by (7) in the laboratory frame assuming $\mathbf{G}_{\perp}[(4)]$ to be known. In this case, constraint (iii) would yield an analytical solution whereas (ii) would not.

\section{Crystal alignment}

In this section, we discuss how these angle-setting calculations may be implemented in a typical surface diffraction experiment starting from unknown crystal and surface orientations. To determine the sample's crystallographic orientation in the laboratory coordinate system requires observation of two or more bulk-diffraction peaks (Busing \& Levy, 1967; Hamilton, 1974). These can be difficult to find if the peaks are very sharp in all directions. Therefore, as pointed out by Vlieg, Van der Veen et al. (1987), it is convenient to begin by determining the physical or cut-surface normal $\mathbf{n}_{p}$ in the laboratory coordinate system. This facilitates accessing crystal truncation rods (Robinson, 1986), which are parallel to $\mathbf{n}_{p}$ and which are useful in locating the bulk diffraction peaks.

The orientation of the cut surface can be determined using specular reflection of X-rays or even relatively long wavelength laser light. After the sample is mounted, the $\varphi$ and $\chi$ circles are adjusted until the reflected beam is independent of rotation about the $\omega$ axis. Suppose this occurs when $\varphi=\varphi_{0}$ and $\chi=\chi_{0}$, we can then calculate the sample surface normal $\mathbf{n}_{p}$ in 
the laboratory coordinate system when all angle settings are zero (initial position):

$$
\mathbf{n}_{p}=\Phi\left(-\varphi_{0}\right) X\left(-\chi_{0}\right) \mathbf{n}_{p}^{\prime},
$$

where

$$
\mathbf{n}_{p}^{\prime}=\left[\begin{array}{l}
0 \\
0 \\
1
\end{array}\right] \text {. }
$$

Using (9) and (10) for the matrices $\Phi$ and $X$, respectively, we obtain

$$
\mathbf{n}_{p}=\left[\begin{array}{c}
\sin \varphi_{0} \cos \chi_{0} \\
-\sin \chi_{0} \\
\cos \varphi_{0} \cos \chi_{0}
\end{array}\right]=\left[\begin{array}{c}
\left(n_{p}\right)_{x} \\
\left(n_{p}\right)_{y} \\
\left(n_{p}\right)_{z}
\end{array}\right] .
$$

Here, $\left(n_{p}\right)_{x}$ and $\left(n_{p}\right)_{y}$ and $\left(n_{p}\right)_{z}$ are the $x, y$ and $z$ components of $\mathbf{n}_{p}$ in the laboratory system.

To orient the sample crystallographically requires searching for two or more bulk reflections. Here, we describe a search procedure based on accessing a point on two different crystal truncation rods of the sample. This discussion serves to illustrate the formalism developed in previous sections. We assume that the sample miscut is small so that the normal to the cut surface and that of the crystallographic plane of interest are approximately parallel, i.e. $\mathbf{n} \simeq \mathbf{n}_{p}$. Furthermore, we assume that two reciprocal-lattice vectors of the sample, $\mathbf{b}_{1}$ and $\mathbf{b}_{2}$, are in this crystallographic plane and that $\mathbf{b}_{3}$ is normal to it. The general reciprocal-lattice vector $\mathbf{G}$ is given by

$$
\mathbf{G}=h \mathbf{b}_{1}+k \mathbf{b}_{2}+l \mathbf{b}_{3},
$$

where $h k l$ are the usual Miller indices except that here $l$ need not be an integer.

We have in our UHV chamber a low-energy electron diffraction system equipped with a twodimensional position-sensitive detector whose diffraction patterns along with knowledge of $\mathbf{n}_{p}$ can aid in locating the crystal truncation rods. Suppose that we have accessed with the X-ray diffractometer in the four-circle mode $(\delta=0)$ a point on each of the crystal truncation rods associated with the reciprocal-lattice vectors $\mathbf{b}_{1}$ and $\mathbf{b}_{2}$. We denote these points $\mathbf{Q}_{1}$ and $\mathbf{Q}_{2}$, corresponding to angle settings $\varphi_{1}, \chi_{1}, \omega_{1}, 2 \theta_{1}^{\prime}$ and $\varphi_{2}, \chi_{2}, \omega_{2}, 2 \theta_{2}^{\prime}$, respectively. When the diffractometer is returned to its initial configuration $(\varphi=\chi=\omega=0)$, these points will be be represented in the laboratory coordinate system by reciprocal-lattice vectors $\mathbf{G}_{1}$ and $\mathbf{G}_{2}$ with Miller indices $\left(10 l_{1}\right)$ and $\left(01 l_{2}\right)$, respectively. Using the expression for $\mathbf{Q}$ in the laboratory coordinate system in (1) with $\delta=0$, we can obtain expressions for the $\mathbf{G}_{i}$ :

$$
\mathbf{G}_{i}=k \Phi\left(-\varphi_{i}\right) X\left(-\chi_{i}\right) \Omega\left(-\omega_{i}\right)\left[\begin{array}{c}
\cos 2 \theta_{i}^{\prime}-1 \\
\sin 2 \theta_{i}^{\prime} \\
0
\end{array}\right],
$$

where the matrices $\Phi, X$ and $\Omega$ are given by (9), (10) and (11). Under the assumption that $\mathbf{n} \simeq \mathbf{n}_{p}$, the $\mathbf{G}_{i}$ can be projected onto the crystallographic plane of interest to yield the $\mathbf{b}_{i}$ in the laboratory frame:

$$
\mathbf{b}_{i} \simeq \mathbf{G}_{i}-\left(\mathbf{n}_{p} \cdot \mathbf{G}_{i}\right) \mathbf{n}_{p}, \quad \text { for } i=1,2 .
$$

In this example, $\mathbf{b}_{3}$ is perpendicular to the crystallographic plane of interest and known to have magnitude $b_{3}$, so

$$
\mathbf{b}_{3}=b_{3} \mathbf{n} \simeq b_{3} \mathbf{n}_{p}
$$

and the Miller indices $l_{i}$ of the points on the truncation rods are

$$
l_{i} \simeq \mathbf{n}_{p} \cdot \mathbf{G}_{i} / b_{3}, \quad \text { for } i=1,2,
$$

where $\mathbf{n}_{p}$ is given by (37). Hence, we have obtained approximations to all of the $\mathbf{b}_{i}$ and, by (38), to any bulk reciprocal-lattice vector $\mathbf{G}$ in the laboratory frame at the initial sample position. Approximate values of $\mathbf{G}_{\perp p}$ and $\mathbf{G}_{\| p}$ can be calculated from (3) and the corresponding orthogonal unit-vector triple $\mathbf{r}, \mathbf{s}, \mathbf{t}$ from (5).

To search for the bulk reflection corresponding to the reciprocal-lattice vector $\mathbf{G}$, we need approximate values for sample rotation angles $\varphi, \chi$ and $\omega$. Computation of these in $\S 4$ requires calculating the components $\mathbf{Q}_{\perp p}$ and $\mathbf{Q}_{\| p}$ of the wave vector $\mathbf{Q}$ corresponding to $\mathbf{G}$ as discussed in $\S 5$. Because we are working in the four-circle mode, we have the fixed-detector-angle constraint $\delta=0$, which allows one to calculate $2 \theta^{\prime}$ from (2) and $\mathbf{Q}$ from (1). A second constraint, on the incident angle $\alpha$, can then be imposed using (21) to obtain $\mathbf{Q}_{\perp_{p}}$ and $\mathbf{Q}_{\| p}[c f$. (26)-(30)] and thus the unit-vector triple $\mathbf{r}^{\prime}, \mathbf{s}^{\prime}, \mathbf{t}^{\prime}$ from (8). Since $\mathbf{r}, \mathbf{s}, \mathbf{t}$ have also been determined, the matrix $T_{3}$ in (17) can be calculated to yield approximate values of $\varphi, \chi$ and $\omega$ from (18)-(20). Once two bulk reflections have been found by this procedure, the crystal orientation in the laboratory frame can be determined (Wang, 1992; Busing \& Levy, 1967; Hamilton, 1974).

\section{Concluding remarks}

A summary of the various suggested modes of operation of the rotating-detector five-circle diffractometer is presented in Table 1. A list of possible constraints appears in the second column where one from each of two sets must be selected. The third column contains derived quantities in the order in which they are calculated in $\S \$ 4$ and 5 .

The method presented here for the calculation of the angle settings of the rotating-detector five-circle diffractometer can also be applied to the conventional four-circle and rotating-table five-circle diffractometers. To apply the method to a four-circle diffractometer, one simply sets $\delta=0$, as shown in the 
Table 1. Summary of operational modes of the rotating-detector five-circle diffractometer

In each mode, one constraint in the second column is imposed from each of types 1 and 2 . Derived quantities are listed in the third column in the order in which they are calculated in the text. $\alpha$ and $\beta$ are the angles of the incident and diffracted beams, respectively, defined in Fig. 4. The angles $\varphi, \chi, \omega$ and $2 \theta^{\prime}$ of the conventional four-circle instrument are defined in Fig. 1. $\delta$ is the detector angle of the five-circle diffractometer defined in Figs. 1 and 2. When $\delta=0$, the diffractometer is identical to the standard four-circle instrument. The wave-vector transfer $\mathbf{Q}$ and its components $\mathbf{Q}_{\perp p}$ and $\mathbf{Q}_{\| p}$ are shown in Fig. 3(b). $\left(Q_{\perp p}\right)_{x},\left(Q_{\perp p}\right)_{y}$ and $\left(Q_{\perp p}\right)_{z}$ are the components of $\mathbf{Q}_{\perp p}$ in the laboratory system. After rotation to the diffraction condition, the normal to the cut surface and crystallographic plane of interest are described by the unit vectors, $\mathbf{n}_{p}^{\prime}$ and $\mathbf{n}^{\prime}$, respectively, as shown in Fig. $4(b)$.

$\begin{array}{ccc}\begin{array}{c}\text { Constraint } \\ \text { type }\end{array} & \text { Constraint } & \text { Derived quantities } \\ 1 & \alpha^{*} & \left(Q_{\perp p}\right)_{x} \\ 1 & \beta^{*} & \left(Q_{\perp p}\right)_{x} \\ 1 & \alpha=\beta \dagger & \left(Q_{\perp p}\right)_{x} \\ 2 & \delta^{*} & 2 \theta^{\prime}, \mathbf{Q},\left(Q_{\perp p}\right)_{y},\left(Q_{\perp p}\right)_{z}, \mathbf{Q}_{\| p}, \varphi, \chi, \omega \\ 2 & \mathbf{n}_{p}^{\prime} \text { horizontal } & \left(Q_{\perp p}\right)_{y},\left(Q_{\perp p}\right)_{z}, \mathbf{Q}, 2 \theta^{\prime}, \delta, \mathbf{Q}_{\| p}, \varphi, \chi, \omega \\ & {\left[\left(n_{p}^{\prime}\right)_{y}=0\right]} & \text { No analytical solution } \\ 2 & \mathbf{n}^{\prime} \text { horizontal } & \\ & {\left[\left(n^{\prime}\right)_{y}=0\right]} & \end{array}$

* Set to any required value.

$\dagger \alpha$ and $\beta$ are determined by the value of $G_{\perp p}$.

preceding section. For the rotating-table five-circle diffractometer, one can define a Cartesian coordinate system fixed to the $\alpha$ circle [see Figs. 1 and 2 in Vlieg, Van der Veen et al. (1987)]. In this coordinate system, all calculations would be the same as for the four-circle diffractometer except that the incident wave vector $\mathbf{K}_{i}$ has both $x$ and $z$ components that depend on the rotation angle $\alpha$.

The angle-setting calculations described in this paper have been implemented in a computer program that has been tested on a rotating-detector five-circle diffractometer at the MATRIX beam line of the NSLS. An earlier version of the program was used to operate the same diffractometer in a four-circle mode for experiments with xenon films physisorbed on a single-crystal Ag(111) surface (Dennison et al., 1992; Wang, 1992; Dai, Angot, Ehrlich, Wang \& Taub, 1993).

This work was partially supported by US National Science Foundation Grants nos. DMR-8704938 and DMR-9011069 and US Department of Energy Grant no. DE-FG02-85ER45183 of the MATRIX Participating Research Team.

\section{APPENDIX \\ Angle calculations for special cases}

\section{Specular scan}

For specular scans, $\mathbf{G}_{\| p}=\mathbf{Q}_{\| p}=0$. Therefore, the total wave-vector transfer equals the perpendicular wave-vector transfer, $\mathbf{Q}=\mathbf{Q}_{\perp p}$. As shown in $\$ \S 3$ and 4 , one must have nonzero values for $\mathbf{G}_{\| p}$ and $\mathbf{G}_{1 p}$ to yield a unique solution for the angle settings. To accomplish this, we find an arbitrary unit vector $\mathbf{r}$ such that

$$
\mathbf{r} \cdot \mathbf{G} / G=0 .
$$

There are an infinite number of choices for $\mathbf{r}$ in the plane perpendicular to $\mathbf{G}$. Similarly, we find a unit vector $r^{\prime}$ such that

$$
\mathbf{r}^{\prime} \cdot \mathbf{Q} / Q=0
$$

An example of $\mathbf{r}^{\prime}$ would be

$$
\mathbf{r}^{\prime}=\left(\mathbf{K}_{i}+\mathbf{Q} / 2\right) /\left|\mathbf{K}_{i}+\mathbf{Q} / 2\right| \text {. }
$$

In this way, we calculate all the angle settings for the specular scan case. Note that the angle settings depend on the choice of $\mathbf{r}$ and $\mathbf{r}^{\prime}$. Thus, there are an infinite number of solutions.

\section{In-plane scan}

In this case, the perpendicular wave-vector transfer $\mathbf{Q}_{\perp p}=\mathbf{G}_{\perp p}=0$. For convenience, we define a dummy unit vector $\mathbf{t}=\mathbf{n}_{p}$. After the sample rotation, this unit vector becomes $t^{\prime}$, which is perpendicular to the scattering plane:

$$
\mathbf{t}^{\prime}=\left(\mathbf{K}_{f} \times \mathbf{K}_{i}\right) /\left|\mathbf{K}_{f} \times \mathbf{K}_{i}\right| .
$$

Using these unit vectors $\mathbf{t}$ and $\mathbf{t}^{\prime}$, we can find a unique solution for the angle settings at a given angle $\delta$ as discussed in $\$ 33$ and 4.

\section{References}

BloCH, J. M. (1985). J. Appl. Cryst. 18, 33-36.

Brennan, S. \& Eisenberger, P. (1984). Nucl. Instrum. Methods, A222, 164-167.

Busing, W. R. \& LevY, H. A. (1967). Acta Cryst. 22, 457-464.

Dai, P., Angot, T., Ehrlich, S. N., Wang, S.-K. \& Taub, H. (1993). In preparation.

Dennison, J. R., Wang, S.-K., Dai, P., Angot, T., Taub, H. \& Ehrlich, S. N. (1993). Rev. Sci. Instrum. 63, 3835-3841.

Feidenhans'L, R. (1989). Surf. Sci. Rep. 10, 105-188.

Hamilton, W. C. (1974). International Tables for X-ray Crystallography, Vol. IV, edited by J. A. Ibers \& W. C. Hamilton, pp. 275-284. Birmingham: Kynoch Press.

Mochrie, S. G. J. (1988). J. Appl. Cryst. 21, 1-3.

Robinson, I. K. (1986). Phys. Rev. B, 33, 3830-3836.

Robinson, I. K. (1988). Aust. J. Phys. 41, 359-367.

RoBINSON, I. K. (1991). Handbook on Synchrotron Radiation, Vol. 3, edited by G. Brown \& D. E. Moncton, pp. 221-266. Amsterdam: Elsevier.

Vlieg, E., Van der Veen, J. F., Macdonald, J. E. \& Miller, M. (1987). J. Appl. Cryst. 20, 330-337.

Vlieg, E., Vant Ent, A., De Jongh, A. P., Neerings, H. \& VAN DER VeEn, J. F. (1987). Nucl. Instrum. Methods, A262, 522-527.

WANG, S.-K. (1992). PhD thesis, Univ. of MissouriColumbia, Missouri, USA.

Warren, B. E. (1969). X-ray Diffraction. Reading, MA: Addison-Wesley. 